\title{
Aperçu
}

\section{Suicides et intoxication aux opioïdes en Alberta (2000-2016)}

\author{
Elaine Y.L. Chan, M.P.H. (1,2); Bruce M. McDonald, M.P.H. (1); Elizabeth Brooks-Lim, M.B.Ch.B., FRCPath (3); \\ Graham R. Jones, Ph. D. (3); Kristin B. Klein, M.D., FRCPC (4); Lawrence W. Svenson, Ph. D. $(1,5,6,7)$
}

Diffuser cet article sur Twitter

\section{Résumé}

Dans le cadre de la crise actuelle des opioïdes au Canada, il est important de s'intéresser au rôle joué par l'intoxication aux opiö̈des dans les décès par suicide et, plus particulièrement, de déterminer si les tendances observées à cet égard sont similaires aux tendances observées pour les décès accidentels. Dans cette analyse, on examine les caractéristiques du suicide par intoxication aux opioïdes et la corrélation entre cette méthode et d'autres moyens de suicide entre 2000 et 2016 en Alberta. La crise des opioïdes ne semble pas avoir causé un nombre disproportionnellement élevé de suicides en Alberta. Les personnes qui décèdent des suites d'une intoxication accidentelle aux opioïdes et celles qui se suicident en s'intoxiquant avec des opioïdes constituent probablement des populations différentes, ce qui nécessite des interventions préventives nuancées en matière de santé publique.

Mots-clés : opioïdes; analgésiques opiö̈des; suicide; surveillance de la santé publique; Canada

\section{Introduction}

Le Canada se retrouve au cœur d'une crise nationale des opioïdes, engendrée en partie par une augmentation de la fréquence de prescription, du dosage et de la puissance de ces drogues depuis les années $1990^{1,2}$ et qui se manifeste par une hausse des taux de méfaits et de décès associés aux opioïdes. En 2016, 2800 décès liés aux opioïdes, et probablement évitables, ont eu lieu au Canada ${ }^{3,4}$. On ne dispose d'aucune donnée à long terme et à l'échelle nationale sur la mortalité associée, mais on sait que le nombre de décès associés aux opioïdes a doublé en Ontario entre 1991 et $2004^{2}$. En dix ans, le taux d'hospitalisation à la suite d'une intoxication aux opioïdes a augmenté de $53 \%$ au Canada ${ }^{4}$.

Même si la majorité des méfaits et décès associés aux opioïdes sont accidentels, ces derniers peuvent également être la cause de décès intentionnels ${ }^{5,7}$. Environ $30 \%$ des hospitalisations survenues à la suite d'intoxications aux opioïdes au Canada en 2016 découlaient de préjudices auto-infligés, en particulier de tentatives de suicide ${ }^{4}$. Par ailleurs, $16 \%$ des décès causés par la consommation d'opioïdes en Ontario entre 2006 et 2008 étaient des suicides ${ }^{5}$.

On sait que les opioïdes sont couramment utilisés comme méthode de suicide (selon une étude menée à Toronto, les opioïdes sont la catégorie de drogue détectée le plus souvent dans les cas de suicides par surdose survenus entre 1998 et 2007, soit dans $30 \%$ des $\operatorname{cas}^{8}$ ), et les personnes qui souffrent de douleur chronique et ont souvent reçu des ordonnances d'opioïdes dans le passé risquent sans doute davantage de se suicider ${ }^{6,9,10}$. On manque cependant d'information à propos de l'utilisation des opioides comme méthode de suicide dans le contexte de la crise des opioïdes au Canada. On ne sait par exemple pas si le nombre de suicides par intoxication à ces

\section{Points saillants}

- Entre 2000 et 2016, environ $20 \%$ des suicides en Alberta ont été causés par une intoxication aux drogues, dont $22 \%$ en lien avec des opioïdes.

- Un plus grand nombre de femmes que d'hommes se sont suicidés par intoxication aux opioïdes et, parmi les suicides par intoxication aux drogues, on a observé une corrélation entre un âge plus avancé et l'utilisation d'opioïdes.

- Le taux moyen de suicide par intoxication aux opioïdes était de 0,61 pour 100000 années-personnes chez les femmes et de 0,57 pour 100000 années-personnes chez les hommes. Il n'y a eu aucune augmentation notable du taux de suicide par intoxication aux opioïdes entre 2000 et 2016 .

- Les opioïdes utilisés le plus souvent pour se suicider étaient la morphine, la codéine, l'hydromorphone et l'oxycodone.

drogues a augmenté et si les tendances en matière d'utilisation comme méthode de suicide sont similaires aux tendances observées dans les cas de décès accidentels causés par les opioïdes. Notre analyse a pour but de remédier au manque de connaissances en la matière au moyen d'un examen des caractéristiques des individus et des types d'opioïdes utilisés comme méthode de suicide par intoxication ainsi que de l'étude des corrélations entre cette

Rattachement des auteurs :

1. Analytics and Performance Reporting Branch, Alberta Ministry of Health, Edmonton (Alberta), Canada

2. Agence de la santé publique du Canada, Ottawa (Ontario), Canada

3. Office of the Chief Medical Examiner, ministère de la Justice et du Solliciteur général de l'Alberta, Edmonton (Alberta), Canada

4. Office of the Chief Medical Officer of Health, Alberta Ministry of Justice and Solicitor General, Edmonton (Alberta), Canada

5. Division of Preventive Medicine, Faculty of Medicine and Dentistry, University of Alberta, Edmonton (Alberta), Canada

6. School of Public Health, University of Alberta, Edmonton (Alberta), Canada

7. Department of Community Health Sciences, Cumming School of Medicine, University of Calgary, Calgary (Alberta), Canada

Correspondance : Larry Svenson, Alberta Health, 10025, avenue Jasper, Edmonton (Alberta) T5J 1S6; tél. : 780-422-4767; courriel : Larry.Svenson@gov.ab.ca 
méthode et les autres méthodes de suicide, en Alberta, sur une période de 17 ans.

\section{Méthodologie}

Nous avons examiné des données dépersonnalisées provenant du bureau de l'état civil de l'Alberta sur les suicides ayant eu lieu dans cette province entre 2000 et 2016. Nous avons obtenu la manière (p. ex. intentionnel/suicide) dont se sont produits ces décès et la méthode utilisée (p. ex. intoxication) grâce aux codes de la Classification statistique internationale des maladies et des problèmes de santé connexes, dixième révision (CIM-10). Nous avons extrait les données relatives aux décès dont la cause initiale était le suicide (codes X60 à X84 et Y87.0) et nous avons considéré comme suicides par intoxication aux drogues les suicides pour lesquels la cause initiale du décès correspondait aux codes X60 à X64. Nous avons ventilé ces données plus en détail à l'aide des codes T40.0 (opium), T40.1 (héroïne), T40.2 (autres opioïdes), T40.3 (méthadone), T40.4 (autres narcotiques synthétiques) et T40.6 (narcotiques, autres et sans précision). Nous avons considéré les décès dont la cause correspondait à ces codes comme des suicides par intoxication aux opioïdes, sans égard à la présence d'autres types de drogues. Nous avons produit des statistiques descriptives et effectué des analyses de régression logistique en utilisant le sexe et l'âge comme variables indépendantes, à la fois pour les suicides par intoxication aux drogues et pour les suicides par intoxication aux opioïdes. Nous avons observé l'évolution des données pour en déceler des tendances et nous en avons évalué la signification statistique à l'aide de tests du chi carré et d'analyse de la variance.

\section{Résultats}

\section{Caractéristiques démographiques et tendances}

Entre 2000 et 2016, 8344 suicides ont eu lieu en Alberta, 2049 (24,5 \%) commis par des femmes et $6295(75,4 \%)$ par des hommes. Si la majorité (80 \%) n'étaient pas associés aux drogues, $20 \%$ des suicides ont été causés par une intoxication aux drogues, dont 52,9\% commis par des femmes. Environ $22 \%$ des suicides par intoxication aux drogues étaient attribuables aux opioïdes, dont 51,4\% commis par des femmes. Dans un modèle tenant compte du sexe et de l'âge, les femmes étaient 5,7 fois plus susceptibles (intervalle de confiance [IC] à $95 \%: 5,02$ à 6,36) de se suicider par intoxication aux drogues que les hommes ( $p<0,0001)$. Cependant, le sexe n'était pas prédictif d'une intoxication aux opioïdes au sein des suicides par intoxication aux drogues.

Les personnes qui se sont enlevé la vie en s'intoxiquant avec des drogues étaient plus âgées que celles qui se sont suicidées en utilisant d'autres méthodes (les âges moyens étaient respectivement de 47,4 ans [écart-type : 14,2] et de 42 ans [écart-type : 17]). Dans un modèle ajusté en fonction du sexe, les taux de suicide par intoxication aux drogues augmentaient de 2,4\% comparativement aux suicides liés à d'autres causes (IC à $95 \%: 2$ à 2,7\%) pour chaque année ajoutée à l'âge $(p<0,0001)$. Les rapports de cotes corrigés selon le sexe des suicides causés par une intoxication aux opioïdes augmentaient également de 0,8 \% parmi les suicides causés par une intoxication aux drogues (IC à $95 \%$ : 0 à 1,7\%) pour chaque année ajoutée à l'âge $(p<0,05)$. Lầge moyen des personnes qui se sont suicidées en s'intoxiquant avec des opioïdes était de 48,8 ans (écart-type : $14,4)$.

La proportion de femmes sur l'ensemble des suicides $(p: 0,35)$, le taux de suicides causés par une intoxication aux drogues $(p: 0,71)$ et le taux de suicides causés par une intoxication aux opioïdes $(p: 0,62)$ sont demeurés relativement constants entre 2000 et 2016. L'âge moyen pour l'ensemble des suicides $(p: 0,063)$, pour les suicides par intoxication aux drogues $(p: 0,36)$ et pour les suicides par intoxication aux opioïdes $(p: 0,43)$ est demeuré stable au cours de ces 17 ans.

\section{Moyens de suicides au fil du temps}

De façon générale, en Alberta, entre 2000 et 2016, les suicides dont la cause n'était pas associée aux drogues étaient plus courants que les suicides par intoxication aux drogues. Chez les femmes, ils ont représenté, en moyenne, $57 \%$ des suicides annuels, et ont été plus nombreux en proportion que les suicides causés par intoxication aux drogues (toutes drogues confondues) pour chaque année de la période examinée, sauf en 2004 et en 2011, où l'intoxication aux drogues s'est révélée la cause de respectivement $53 \%$ et $55 \%$ des suicides. Le taux annuel moyen de suicides non associés à l'utilisation de drogues chez les femmes était de 3,9 pour 100000 années-personnes. Chez les hommes, les méthodes de suicide autres que le recours aux drogues étaient prédominantes, les suicides non causés par l'intoxication aux drogues représentant en moyenne $88 \%$ des suicides annuels, avec un taux annuel moyen de 17,9 pour 100000 années-personnes. Le taux moyen de suicides causés par une intoxication à des drogues autres que les opioïdes était légèrement plus élevé chez les femmes que chez les hommes (2,3 contre 1,9 pour 100000 années-personnes) et cette méthode était associée à un pourcentage moyen beaucoup plus élevé de suicides chez les femmes que chez les hommes (34 \% contre $10 \%$ ) (figure 1). Parallèlement, le taux moyen de suicides par intoxication aux opioïdes pour 100000 annéespersonnes était de 0,61 chez les femmes et de 0,57 chez les hommes, et le pourcentage annuel moyen de suicides attribuables à une intoxication aux opioïdes de $9 \%$ chez les femmes et de $3 \%$ chez les hommes.

Les méthodes de suicide variaient davantage chez les femmes que chez les hommes, mais le taux de suicide global variait davantage chez les hommes (figure 1). Chez les femmes, à partir de 2014, on a observé une tendance à la baisse du nombre de suicides causés par une intoxication à des drogues autres que les opioïdes jusqu'à des taux jamais observés auparavant, ainsi que des tendances à la hausse simultanées du nombre de suicides non attribuables à l'utilisation de drogues et du nombre de suicides associés à une intoxication aux opioïdes. Chez les hommes, le taux et la contribution en pourcentage associés aux suicides causés par une intoxication aux opioïdes ont également augmenté, pour culminer en 2015.

\section{Types d'opioïdes utilisés au fil du temps dans les suicides liés à ces drogues}

Chez les femmes comme chez les hommes, les opioïdes les plus couramment utilisés comme méthode de suicide ont été surtout la morphine, la codéine, l'hydromorphone et l'oxycodone (figure 2). Le deuxième type d'opioïdes le plus couramment utilisé relève de la catégorie des " autres narcotiques synthétiques ", qui comprend le fentanyl et l'U-47700. Au début des années 2000 (pour les deux sexes), en 2013 (chez les hommes) et en 2014 (chez les femmes), on a observé une augmentation, en proportion, de l'utilisation d'" autres narcotiques synthétiques » et une diminution, en 
FIGURE 1

Taux de suicide (globaux) et méthodes de suicide (taux et proportions), Alberta, 2000-2016, selon le sexe
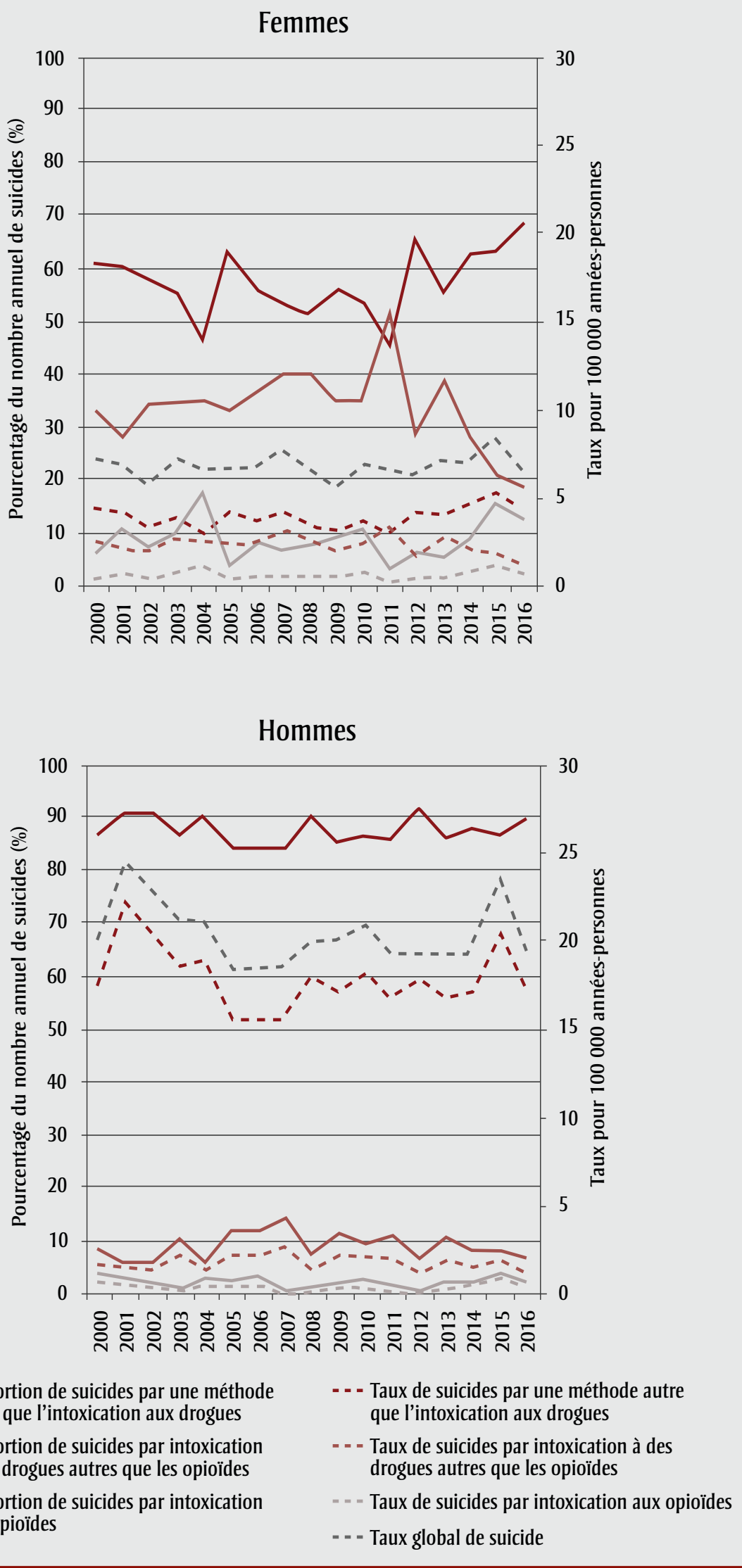

proportion également, de l'utilisation d'« autres opioïdes » comme méthode de suicide.

\section{Analyse}

En Alberta, les personnes qui sont décédées de mort accidentelle causée par une intoxication aux opioïdes en 2016 étaient en majorité des hommes (73\%) et étaient âgées de 30 à 39 ans $^{11}$. Pour les décès par suicide, on a observé des taux en lien avec une intoxication aux opioïdes légèrement plus élevés chez les femmes, avec un âge moyen de 49 ans. Ces données indiquent que les décès accidentels et les suicides causés par une intoxication aux opioïdes surviennent au sein de populations distinctes, et qu'il s'agit donc vraisemblablement de deux enjeux différents en matière de santé publique. Les résultats de précédentes recherches ont également fait état de davantage de suicides causés par une intoxication aux opioïdes chez les femmes et de davantage de décès causés par l'abus d'opioïdes chez les hommes ${ }^{12}$.

On n’a observé aucune hausse significative du taux de suicide par intoxication aux opioïdes entre 2000 et 2016 qui soit similaire à celle observée pour les décès accidentels en Alberta entre 2011 et $2016^{7}$, et les caractéristiques démographiques relatives au nombre de suicides n'ont pas non plus changé de façon significative au fil du temps. Les variations de taux relatives aux méthodes employées et la stabilité du taux global de suicides chez les femmes conduisent à envisager des substitutions dans les méthodes de suicide plutôt que des changements de fréquence dans les suicides eux-mêmes. L'augmentation du taux de suicides causés par une intoxication aux opioïdes vers 2014 et 2015 pourrait découler d'un meilleur accès à des opioïdes synthétiques comme le fentanyl.

Les tendances de consommation liées aux suicides par intoxication aux opioïdes sont différentes de celles observées dans les décès accidentels causés par une intoxication aux opioïdes : la consommation de fentanyl est la principale cause de mort accidentelle associée aux opioïdes ${ }^{7}$, alors que ce sont d'autres opioïdes (la morphine, la codéine, l'hydromorphone et l'oxycodone) qui sont largement utilisés comme méthodes de suicide. Si on tient compte également des différences dans les caractéristiques démographiques, on peut vraisemblablement conclure que les suicides et 
FIGURE 2

Types d'opioïdes utilisés dans les suicides par intoxication à ces drogues, Alberta, 2000-2016, selon le sexe

\section{Femmes}

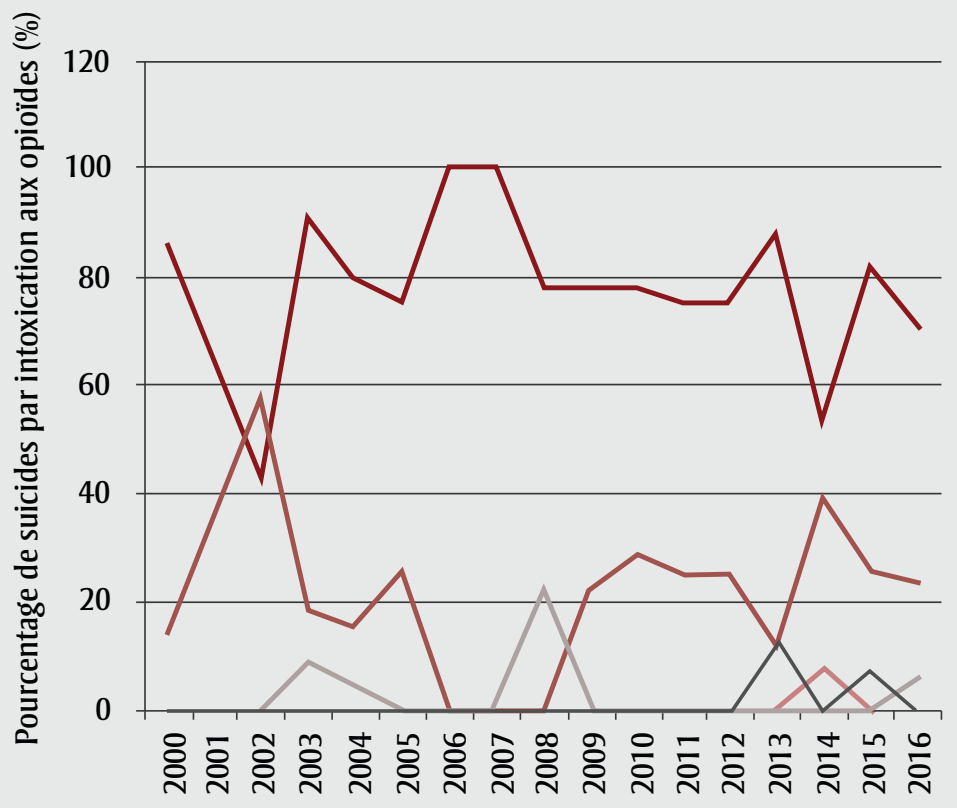

Hommes
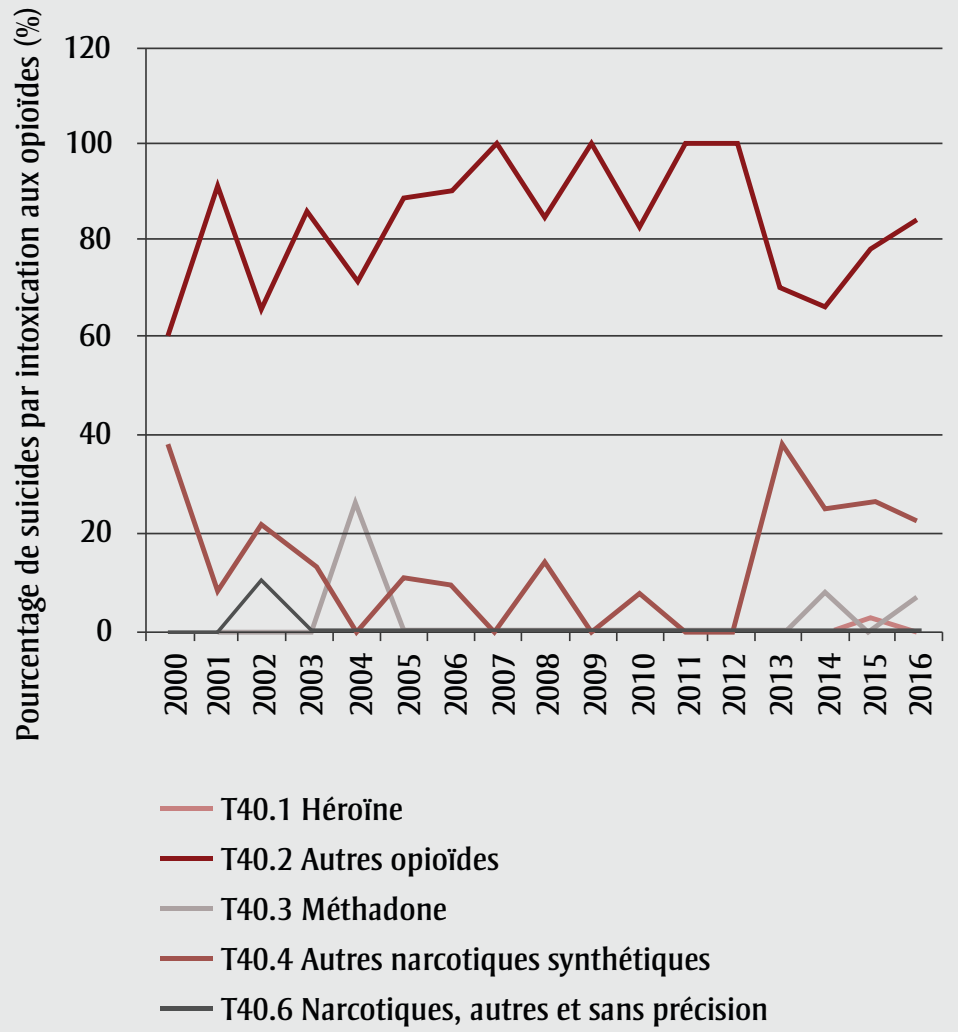

Notes : On a utilisé les codes de la Classification statistique internationale des maladies et des problèmes de santé connexes, dixième révision (CIM-10) afin de définir les types d'opioïdes. L'opium (T40.0) n'a pas été utilisé dans les suicides en Alberta entre 2000 et 2016. Les « autres opioïdes» (T40.2) sont la morphine, la codéine, l'hydromorphone et l'oxycodone. Les « autres narcotiques synthétiques » (T40.4) sont les opioïdes synthétiques comme le fentanyl et l'U-47700. les décès accidentels associés aux opioïdes se produisent en majorité au sein de groupes de population différents en Alberta, qui ont des accès différents à divers types d'opioïdes.

\section{Points forts et limites}

Parmi les points forts de cette étude, on compte l'utilisation des données du bureau de l'état civil de l'Alberta, qui a permis de consigner tous les suicides survenus dans la province pendant 17 ans. Toutefois, seuls les suicides dont la cause principale était une intoxication aux drogues ont été considérés comme tels : nous n'avons pas tenu compte des suicides dont la cause principale était l'emploi d'une autre méthode (p. ex. pendaison, arme à feu) mais avec présence d'une intoxication aux drogues. De plus, le recours aux codes de la CIM-10 ne permet pas de déterminer le rôle de certains opioïdes, limitant ainsi le degré de précision de notre étude à celui de ces codes. Par ailleurs, le rôle des opioïdes dans les décès accidentels causés par une intoxication aux drogues peut avoir été sous-estimé, particulièrement durant les premières années, étant donné que les pratiques de classification ne permettent pas toujours d'indiquer de façon précise les drogues en cause dans une intoxication. L'utilisation de données administratives recueillies sur une longue période peut porter à confusion car les différences observées pourraient découler de changements apportés aux pratiques de classification au fil du temps à la suite de crises connues plutôt que de véritables différences.

\section{Conclusion}

En Alberta, les décès accidentels et les suicides causés par une intoxication aux opioïdes se distinguent à la fois par les caractéristiques démographiques, les tendances observées et les types d'opioïdes utilisés, ce qui conduit à envisager qu'il s'agit là de phénomènes différents au sein de la crise des opioïdes. La prévention du suicide par intoxication aux opioïdes exigera probablement une intervention en santé publique distincte de celle actuellement employée pour prévenir principalement les décès causés par la consommation de fentanyl illicite et les décès accidentels causées par une intoxication aux opioïdes.

\section{Remerciements}

Les auteurs tiennent à remercier Sean L'Heureux et le bureau de l'état civil de l'Alberta de leur soutien. 


\section{Conflits d'intérêts}

Les auteurs déclarent n'avoir aucun conflit d'intérêts.

\section{Contribution des auteurs et avis}

EC, BM et LS ont conçu et mis au point l'étude. BM a extrait et dépersonnalisé les données pour l'analyse et EC a effectué l'analyse. Tous les auteurs ont participé à la rédaction du manuscrit et à l'interprétation des données. Ils ont tous lu et approuvé le manuscrit final.

Le contenu de cet article et les opinions qui y sont exprimées n'engagent que les auteurs et ne sont pas forcément représentatifs de la position du Gouvernement du Canada.

\section{Références}

1. King NB, Fraser $V$, Boikos $C$, et al. Determinants of increased opioidrelated mortality in the United States and Canada, 1990-2013: a systematic review. Am J Public Health. 2014; 104(8):e32-e42. doi: 10.2105/AJPH.2014 .301966 .

2. Dhalla IA, Mamdani MM, Sivilotti MLA, et al. Prescribing of opioid analgesics and related mortality before and after the introduction of long-acting oxycodone. CMAJ. 2009;181(12):891896. doi: $10.1503 / \mathrm{cmaj} .090784$.

3. Comité consultatif spécial sur l'épidémie de surdoses d'opioïdes. Rapport national : Décès apparemment liés à la consommation d’opioïdes (décembre 2017) [Internet]. Ottawa (Ont.) : Agence de la santé publique du Canada; 2017 [consultation le 15 janv. 2018]. En ligne à : https://www.canada .ca/fr/sante-publique/services /publications/vie-saine/deces -apparemment-lies-consommation -opioides-rapport-2016-2017-decembre .html

4. Institut canadien d'information sur la santé (ICIS). Les « importantes » répercussions de la crise des opioïdes sur les systèmes de santé canadiens [Internet]. Ottawa (Ont.) : ICIS; 2017. En ligne à : https://www.cihi.ca/fr/les -importantes-repercussions-de-la-crise -des-opioides-sur-les-systemes-de-sante -canadiens
5. Madadi P, Hildebrandt D, Lauwers AE, et al. Characteristics of opioid-users whose death was related to opioidtoxicity: a population-based study in Ontario, Canada. PLoS ONE. 2013; 8(4):e60600. doi: 10.1371/journal.pone 0060600 .

6. Madadi P, Persaud N. Suicide by means of opioid overdose in patients with chronic pain. Curr Pain Headache Rep. 2014;18(11):460. doi: 10.1007 /s11916-014-0460-1.

7. Alberta Health Analytics and Performance Reporting Branch. Opioids and substances of misuse, Alberta report, 2017 Q3. Edmonton (AB) : Government of Alberta; 2017. En ligne à : https:// open.alberta.ca/dataset/1cfed7da-2690 -42e7-97e9-da175d36f3d5/resource /e69ffcb0-6d34-4e2b-bf35-7d13ec35de59 /download/Opioids-Substances-Misuse -Report-2017-Q3.pdf

8. Sinyor M, Howlett A, Cheung AH, et al. Substances used in completed suicide by overdose in Toronto: an observational study of coroner's data. Can J Psychiatry. 2012;57(3):184-91. En ligne à : http://journals.sagepub.com/doi /abs/10.1177/070674371205700308

9. Deyo RA, Von Korff M, Duhrkoop D. Opioids for low back pain. BMJ. 2015; 350:g6380. doi: 10.1136/bmj.g6380.

10. Demidenko MI, Dobscha SK, Morasco $\mathrm{BJ}$, et al. Suicidal ideation and suicidal self-directed violence following clinician-initiated prescription opioid discontinuation among long-term opioid users. Gen Hosp Psychiatry. 2017;47: 29-35. doi: 10.1016/j.genhosppsych.2017 .04 .011 .

11. Comité consultatif spécial sur l'épidémie de surdoses d'opioïdes. Rapport national : Décès apparemment liés à la consommation d'opioïdes au Canada (janvier 2016 à mars 2017) [Internet]. Ottawa (Ont.) : Agence de la santé publique du Canada; 2017. En ligne à : https://www.canada.ca/fr/sante -publique/services/publications/vie -saine/deces-apparemment-lies -consommation-opioides-rapport -2016.html

12. Caupp S, Steffan J, Shi J, et al. Opioid drug poisonings in Ohio adolescents and young adults, 2002-2014. Clin Toxicol (Phila). 2018;Jan 11:1-8. doi: 10.1080/15563650.2018.1424889. 